\title{
Desenvolvimento pós-natal e potencial teratogênico da prole de ratos Wistar no estudo da toxicidade reprodutiva de duas preparações fitoterápicas contendo soja Glycine max (L.) Merr
}

[Neonatal development and teratogenic potential of Wistar rats offspring in the study of reproductive toxicity of two commercial phytotherapic preparations with soy Glycine max (L.) Merr]

\author{
C.B. Hollenbach ${ }^{1}$, C.E. Bortolini ${ }^{1}$, J.M. Batista ${ }^{1}$, E.B. Hollenbach ${ }^{1}$, T.L. Schuch ${ }^{1}$, M.H. Pacheco ${ }^{1}$, \\ F.B. Mello ${ }^{2}$, J.R. Mello ${ }^{1}$ \\ ${ }^{1}$ Instituto de Ciências Básicas da Saúde - UFRGS \\ Av. Sarmento Leite, 500 \\ 90046-900 - Porto Alegre, RS \\ ${ }^{2}$ Universidade Estadual do Rio Grande do Sul - Porto Alegre, RS
}

\begin{abstract}
RESUMO
Avaliou-se o efeito de duas preparações fitoterápicas comerciais que continham soja sobre o desenvolvimento geral e sexual da progênie de ratos Wistar e sobre o potencial teratogênico das preparações fitoterápicas durante um estudo de toxicidade reprodutiva. Para tanto, observaram-se, diariamente, as características de desenvolvimento dos filhotes e analisou-se o comportamento em campo aberto, e, para avaliar o potencial teratogênico, realizou-se diafanização dos fetos removidos por cesariana. Inferiu-se que o tratamento dos pais não comprometeu o desenvolvimento de sua progênie e também não determinou efeitos teratogênicos aos fetos de ratos Wistar.
\end{abstract}

Palavras-chave: rato, soja, fitoterápicos, toxicidade reprodutiva

\begin{abstract}
The effect of two commercial phytotherapic preparations containing soy was evaluated on the general and sexual development of Wistar rats offspring and on the teratogenic potential during a reproductive toxicity study. For this, the offspring development characteristics were daily observed, and the behavior in open field was studied. To assess the teratogenic potential, diaphanization of the fetuses removed by caesarean section was done. It was concluded that the treatment of the parents did not compromise the development of their progeny and also did not determine the teratogenic effects in the fetuses.
\end{abstract}

Keywords: rat, soy, phytotherapic, reproductive toxicology

\section{INTRODUÇÃO}

A soja, leguminosa da família Fabaceae, é cultivada como alimento tanto para humanos quanto para animais. O grão é rico em substâncias chamadas isoflavonas, que, por sua semelhança estrutural ao estrógeno humano, determinam efeitos estrogênicos importantes, sendo, por isso, referido como fitoestrógenos (Wang e Murphy, 1994).

Há registros na literatura referentes ao uso da soja e de suas isoflavonas para a prevenção e o tratamento de enfermidades. Além da substituição do leite animal em crianças com manifestações alérgicas, as principais indicações terapêuticas são: tratamento de sintomas pré e pós-menopausa na prevenção da osteoporose, na prevenção e no tratamento de câncer, na prevenção de doenças cardiovasculares e como auxiliar no tratamento dos diabetes.

Os testes de avaliação da toxicidade reprodutiva geralmente compreendem a exposição de animais sexualmente maduros antes da concepção, durante o desenvolvimento pré-natal e após o nascimento e, continuadamente até sua maturação sexual (Lemônica, 2001). Os estudos mais utilizados para essas avaliações são

Recebido em 4 de novembro de 2008

Aceito em 16 de julho de 2010

E-mail: clarissa.hollenbach@gmail.com

Apoio: CNPq e CAPES 
divididos em três segmentos e são adaptados de normas da Environmental Protection Agency e recomendados pela Food and Drugs Administration e Organization for Economic Cooperation and Development (Organization..., 1998).

Nas fases pré e pós-natal, os órgãos sexuais e o sistema nervoso central que ainda estão se diferenciando podem ser atingidos por substâncias presentes no sangue materno através da placenta, durante a gestação, ou através do leite, durante a lactação. Além disso, indivíduos expostos durante períodos críticos de desenvolvimento são mais vulneráveis à ação de substâncias químicas em função da menor capacidade metabólica e excretora e da ausência de muitos mecanismos de retroalimentação do sistema endócrino (Zenick e Klegg, 1989). O tratamento dos machos antes da concepção pode resultar em infertilidade, morte embrionária e anomalias não específicas, bem como em redução do peso ao nascer, diminuição do tamanho da ninhada e retardo no desenvolvimento pós-natal (Colie, 1993; Delclos et al., 2001).

As características do concepto e sua capacidade de desenvolvimento e maturação são expressões da capacidade de fertilização de células germinativas, concepção bem-sucedida e o subsequente desenvolvimento intrauterino. $\mathrm{O}$ desenvolvimento peri e pós-natal depende do potencial de desenvolvimento do indivíduo e de sua habilidade de enfrentar variadas circunstâncias ambientais (Mello e Langeloh, 2006).

O objetivo do trabalho foi avaliar, na progênie de ratos e ratas tratados durante o período reprodutivo, o efeito de duas preparações fitoterápicas comerciais contendo soja sobre o desenvolvimento geral e sexual dos filhotes e sobre o potencial teratogênico das preparações.

\section{MATERIAL E MÉTODOS}

Os fitoterápicos utilizados nos experimentos e a isoflavona usada no controle positivo foram adquiridos em estabelecimento farmacêutico local, com os mesmos números de lote, data de fabricação e dentro do prazo de validade. Foram utilizados os fitoterápicos Soyfemme® (Aché Laboratórios Farmacêuticos S.A.) e Isoflavine® (Herbarium Laboratório Botânico Ltda.) As composições declaradas na bulas dos medicamentos foram: Extrato seco de Glycine $\max$ (L.) Merr $40 \%$ em cápsula de 150mg (Soyfemme®) e Extrato de Glycine max (L.) Merr $40 \%$ em cápsula de $75 \mathrm{mg}$ (Isoflavine $®)$.

As formulações fitoterápicas foram obtidas por meio da diluição do conteúdo das cápsulas dos medicamentos, utilizando-se como veículo água destilada. A dosagem inicial foi obtida segundo as recomendações dos fabricantes. A dose terapêutica foi $4,3 \mathrm{mg} / \mathrm{kg}$ e a dose de Isoflavine ${ }^{\circledR}$ foi ajustada para se igualar à dose de Soyfemme®. As dosagens subsequentes foram calculadas para cinco e 10 vezes a dose recomendada pelo fabricante. A formulação usada no grupo-controle positivo foi obtida mediante diluição da isoflavona em água destilada, na dose de $4 \mathrm{mg} / \mathrm{kg}$.

Foram utilizados ratos Wistar, machos e fêmeas com 120 dias de idade, provenientes do Centro de Reprodução e Experimentação de Animais de Laboratório da Universidade Federal do Rio Grande do Sul (UFRGS). Durante o experimento, os animais, mantidos em biotério sob condição constante de umidade $(50 \% \pm 5)$, temperatura $\left(21^{\circ} \mathrm{C} \pm 2\right)$ e ciclo de luz claro/escuro de 12 horas, foram alimentados com ração comercial (Nuvital CR 1, Nuvital, Colombo, PR) e água ad libitum, durante todo o período experimental. $\mathrm{O}$ alojamento, $\mathrm{o}$ manejo, $\mathrm{o}$ tratamento e a eutanásia obedeceram às normas publicadas pelo Colégio Brasileiro de Experimentação Animal, obedecendo às leis brasileiras, com aprovação do Comitê de Ética em Pesquisa da UFRGS.

Foram formados oito grupos experimentais, constituídos de 32 animais cada (oito machos e 24 fêmeas): seis grupos (G) teste receberam $4,3 \mathrm{mg} / \mathrm{kg}, \quad 21,5 \mathrm{mg} / \mathrm{kg}$ e $43 \mathrm{mg} / \mathrm{kg}$ das formulações fitoterápicas Soyfemme ${ }^{\circledR}$ (GS) e Isoflavine ${ }^{\circledR}(\mathrm{GI})$, formando: GS1, GS2, GS3 e GI1, GI2, GI3, respectivamente. O grupocontrole negativo (GC-) foi tratado com o veículo (água destilada) e o grupo-controle positivo $(\mathrm{GC}+)$ recebeu isoflavonas de soja, na dose $4 \mathrm{mg} / \mathrm{kg}$, completando os grupos experimentais. Os animais foram tratados diariamente, por via oral, com sonda orogástrica, antes e durante o acasalamento, a gestação e a lactação. O acasalamento teve duração de 21 dias; na caixa de cada um dos machos foram 
introduzidas três fêmeas virgens, permanecendo com eles duas horas por dia (das $7 \mathrm{~h}$ às $9 \mathrm{~h}$ ), correspondendo ao final do período escuro.

Metade das fêmeas prenhes foi submetida à cesariana no $21^{\circ}$ dia de gestação. Após a eutanásia, o útero foi incisado longitudinalmente com tesoura, em toda sua extensão. Os fetos foram retirados e observados quanto à vitalidade e à presença de malformações macroscópicas externas. Após a retirada dos fetos, o útero foi inspecionado e foram contados os implantes uterinos. Os fetos foram fixados em formalina tamponada (10\%) e processados conforme técnica de coloração óssea com alizarina, como descrito por Taylor e Van Dike (1985). A avaliação individual dos fetos foi realizada em lupa com aumento de quatro vezes, tendo como parâmetro de avaliação o Atlas de Anomalias Externas e Esqueléticas de Ratos (Chahould e Faqi, 1997). O restante das fêmeas prenhes pariu a termo, e suas proles foram avaliadas até a manifestação das características sexuais, nos machos (descida dos testículos à bolsa escrotal e separação prepucial) e nas fêmeas (abertura do canal vaginal), e até a realização do teste de comportamento em campo aberto, aos 75 dias de idade.

O desenvolvimento pós-natal da prole das fêmeas paridas foi observado desde o dia do nascimento (dia zero). As ninhadas foram pesadas diariamente até o dia 36 pós-natal, e os filhotes individualmente nos dias zero, sete, 14, e 21. Cada filhote foi avaliado quanto ao desenvolvimento físico geral (dados não apresentados), no qual foi observado o dia do aparecimento da penugem, o descolamento do pavilhão auricular, a erupção dos dentes incisivos, o desenvolvimento dos pelos, a abertura dos olhos e o desenvolvimento sexual, quando foram observados o dia da descida dos testículos, a separação prepucial e a abertura do canal vaginal.

O comportamento da prole foi testado por meio do teste de campo aberto e consistiu na mensuração das variáveis comportamentais dos filhotes aos 75 dias de idade. Foram analisadas as categorias comportamentais: atividade exploratória - caracterizada pela locomoção total e permanência no centro -, "escanear" e "rearing" - ato de permanecer sobre as duas patas traseiras para investigar o ambiente, no centro do campo aberto ou nas laterais, respectivamente -, e autolimpeza - número de vezes em que o animal utiliza as patas dianteiras para limpeza (Azevedo, 2005).

As variáveis quantitativas foram submetidas à análise da variância (ANOVA), e as variáveis qualitativas foram analisadas pelo teste quiquadrado. $\mathrm{O}$ nível de significância foi estabelecido para uma confiança de $99 \%$ $(\mathrm{P}<0,01)$ para o teste qui-quadrado e de $95 \%$ $(\mathrm{P}<0,05)$ para o teste $\mathrm{F}$ (Laponi, 2000).

\section{RESULTADOS}

$\mathrm{Na}$ Tab. 1, apresentam-se os índices reprodutivos de ratas tratadas com Soyfemme ${ }^{\circledR}$ em três doses, $4,3,21,5$ e $43 \mathrm{mg} / \mathrm{kg}$, e com Isoflavine em duas doses, 4,3 e $21,5 \mathrm{mg} / \mathrm{kg}$, e de ratas dos grupos controle negativo e controle positivo que pariram a termo. No GI3, não houve partos. Quanto ao número de filhotes por ninhada e à massa corporal dos filhotes, não houve diferença significativa entre os grupos (ANOVA; P>0,05). Taxas de parto, de viabilidade e de desmama em GS2, taxa de desmame em GS3 e taxas de parto e de natalidade em GI2 foram mais baixas em relação às taxas obtidas em GC- (teste $\chi^{2}$; $\mathrm{P}<0,01)$.

Tabela 1. Índices reprodutivos de ratas tratadas com Soyfemme ${ }^{\circledR}$ (GS), em três doses, e com Isoflavine ${ }^{\circledR}(\mathrm{GI})$, em duas doses, e de ratas dos grupos controle positivo (GC+) e controle negativo (GC-) que pariram a termo (valores médios $\pm \mathrm{EPM}$ )

\begin{tabular}{|c|c|c|c|c|c|c|c|}
\hline Índice reprodutivo & GC- & $\mathrm{GC}+$ & $\begin{array}{c}\mathrm{GS} 1 \\
(4,3 \mathrm{mg} / \mathrm{kg})\end{array}$ & $\begin{array}{c}\mathrm{GS} 2 \\
(21,5 \mathrm{mg} / \mathrm{kg})\end{array}$ & $\begin{array}{c}\mathrm{GS} 3 \\
(43 \mathrm{mg} / \mathrm{kg})\end{array}$ & $\begin{array}{c}\text { GI1 } \\
(4,3 \mathrm{mg} / \mathrm{kg})\end{array}$ & $\begin{array}{c}\mathrm{GI} 2 \\
(21,5 \mathrm{mg} / \mathrm{kg})\end{array}$ \\
\hline Progenitoras (n)/filhotes & $7 / 47$ & $1 / 8$ & $5 / 36$ & $2 / 13$ & $2 / 15$ & $4 / 13$ & $1 / 9$ \\
\hline Filhotes (n)/ninhada & $10,2 \pm 1,6$ & 8 & $10,2 \pm 0,8$ & $7,3 \pm 1,8$ & $7,5 \pm 1,8$ & $5,3 \pm 1,5$ & 9 \\
\hline $\begin{array}{l}\text { Massa corporal dos } \\
\text { filhotes }(\mathrm{g})\end{array}$ & $5,6 \pm 0,14$ & $5 \pm 0,01$ & $6 \pm 0,2$ & $5,4 \pm 0,3$ & $6 \pm 0,5$ & $5,3 \pm 0,1$ & $6 \pm 0,3$ \\
\hline Taxa de parto & $100 \%$ & $100 \%$ & $100 \%$ & $50 \% *$ & $100 \%$ & $100 \%$ & $50 \% *$ \\
\hline Taxa de natalidade & $100 \%$ & $100 \%$ & $87,5 \%$ & $83 \%$ & $100 \%$ & $100 \%$ & $55,5 \% *$ \\
\hline Taxa de viabilidade & $100 \%$ & $100 \%$ & $97,7 \%$ & $60 \% *$ & $100 \%$ & $95,5 \%$ & $100 \%$ \\
\hline Taxa de desmame & $98,4 \%$ & $100 \%$ & $95,3 \%$ & $0 \% *$ & $61,5 \% *$ & $90,9 \%$ & $100 \%$ \\
\hline
\end{tabular}


$\mathrm{Na}$ Tab. 2, encontram-se os índices reprodutivos de ratas tratadas com Soyfemme ${ }^{\circledR}$ em duas diferentes doses, 4,3 e $21,5 \mathrm{mg} / \mathrm{kg}$, e com Isoflavine ${ }^{\circledR}$ (GI) em uma dose, $4,3 \mathrm{mg} / \mathrm{kg}$, e de ratas do grupo-controle negativo, que foram submetidas à cesariana no $21^{\circ}$ dia da gestação. No outros grupos, GC+, GS3, GI2 e GI3, o número de animais não foi suficiente para a realização da cesariana. A massa corporal dos fetos no momento da cesariana e o número de fetos por ninhada não diferiram entre os grupos (ANOVA; P>0,05). O número de filhotes em GS2 e em GI1 e a massa do útero gravídico em GS2 foram diferentes dos demais grupos (ANOVA; $\mathrm{P}<0,05$ ).

Tabela 2. Índices reprodutivos de ratas tratadas com Soyfemme ${ }^{\circledR}$ (GS) em duas doses, e com Isoflavine ${ }^{\circledR}$ (GI), em uma dose, e de ratas do grupo-controle negativo (GC-) que foram submetidas à cesariana no dia 21 de gestação (valores médios \pm EPM)

\begin{tabular}{lcccc}
\multicolumn{1}{c}{ Índice reprodutivo } & \multirow{2}{*}{ GC- } & GS1 & GS2 & GI1 \\
& & $(4,3 \mathrm{mg} / \mathrm{kg})$ & $(21,5 \mathrm{mg} / \mathrm{kg})$ & $(4,3 \mathrm{mg} / \mathrm{kg})$ \\
\hline Progenitoras (n)/filhotes & $(5) 61$ & $(8) 69$ & $(3) 22^{*}$ & $(2) 17^{*}$ \\
Massa do útero grávido (g) & $68,35 \pm 8,0$ & $74,24 \pm 4,6$ & $33,05 \pm 9,2^{*}$ & $64,21 \pm 3,6$ \\
Fetos (n)/ninhada & $10,2 \pm 1,6$ & $10,2 \pm 0,8$ & $7,3 \pm 1,8$ & $10,2 \pm 0,8$ \\
Massa corporal dos fetos (g) & $4,8 \pm 0,1$ & $5,11 \pm 0,05$ & $3,5 \pm 0,8$ & $5,17 \pm 0,6$ \\
\hline
\end{tabular}

*Diferença significativa em relação ao grupo-controle negativo $(\mathrm{P}<0,05)$.

$\mathrm{Na}$ Tab. 3, mostra-se a ocorrência de anormalidades esqueléticas nos fetos de ratas tratadas, durante os períodos de préacasalamento, acasalamento e gestação, com Soyfemme® em duas doses, 4,3 e $21,5 \mathrm{mg} / \mathrm{kg}$, e com Isoflavine ${ }^{\circledR}$ em uma dose, $4,3 \mathrm{mg} / \mathrm{kg}$, e de ratas do grupo-controle negativo. São apresentadas variações e retardos na ossificação de ossos do crânio, tórax, região lombar e membros. Não foram observadas diferenças significativas nas alterações ósseas macroscópicas externas nos fetos entre os grupos tratados e controle negativo (teste $\chi^{2} ; \mathrm{P}>0,01$ ).

Tabela 3. Ocorrência de anormalidades esqueléticas em fetos de ratas tratadas com Soyfemme® (GS), em duas doses, e com Isoflavine ${ }^{\circledR}$ (GI), em uma dose, e de ratas do grupo-controle negativo (GC-)

\begin{tabular}{lcccc}
\multicolumn{1}{c}{ Anormalidade } & GC- & $\begin{array}{c}\text { GS1 } \\
(4,3 \mathrm{mg} / \mathrm{kg})\end{array}$ & $\begin{array}{c}\mathrm{GS} 2 \\
(21,5 \mathrm{mg} / \mathrm{kg})\end{array}$ & $\begin{array}{c}\text { GI1 } \\
(4,3 \mathrm{mg} / \mathrm{kg})\end{array}$ \\
\hline Fetos examinados (n) & 59 & 40 & 27 & 17 \\
Fetos com anormalidades esqueléticas (\%) & 6 & 5 & 4 & 3 \\
Fetos com anomalias (\%) & & & & 0 \\
$\quad$ Membros anteriores pobremente ossificados & 0 & 0 & 0,89 & 1,11 \\
$\quad$ Parietais com ossificação incompleta & 0 & 0,94 & 0 & 0 \\
$\quad$ Supraoccipitais com ossificação incompleta & 1,12 & 0 & 1,20 & 0 \\
$\quad$ Esterno com ossificação incompleta & 3,13 & 0,86 & 2,11 & 0 \\
Malformações & & & & 0 \\
$\quad$ Costela rudimentar & 0 & 0,95 & 0,80 & 1,00 \\
$\quad$ Costelas onduladas & 0 & 1,13 & 0 & 0 \\
$\quad$ Agenesia de costelas & 0 & 1,12 & 0 & 0,89 \\
$\quad$ Esterno de forma irregular & 1,21 & 0 & 0 & 0 \\
Vértebra de forma irregular & 0,44 & 0 & 0 &
\end{tabular}

Na Fig. 1, apresenta-se o desenvolvimento ponderal das progênies de ratas tratadas com Soyfemme ${ }^{\circledR}$ em duas doses, 4,3 e $43 \mathrm{mg} / \mathrm{kg}$, e com Isoflavine ${ }^{\circledR}$ em duas doses, 4,3 e $21,5 \mathrm{mg} / \mathrm{kg}$, e de ratas do grupo-controle negativo, durante o período prévio ao acasalamento, no acasalamento e durante a gestação e a lactação. A progênie foi acompanhada desde o nascimento até o $36^{\circ}$ dia. Verificou-se que não houve diferença entre os grupos tratados e o grupo-controle negativo (ANOVA; $\mathrm{P}>0,05$ ). 

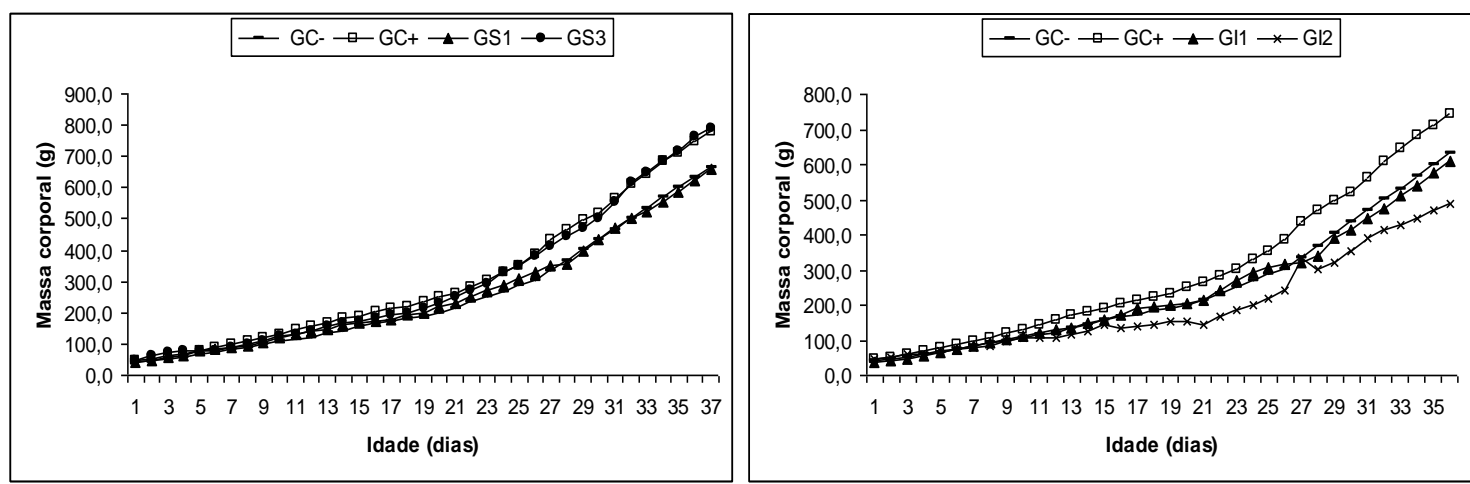

Figura 1. Desenvolvimento ponderal ( $\mathrm{g}$ ) da progênie, desde o nascimento até o $36^{\circ}$ dia, de ratas tratadas com Soyfemme (GS) em duas doses, 4,3 e 43mg/kg, e Isoflavine (GI) em duas doses, 4,3 e 21,5mg/kg, e de ratas dos grupos controle negativo (GC-) e controle positivo $(\mathrm{GC}+)$, durante os períodos de préacasalamento, acasalamento, gestação e lactação.

Na Fig. 2, encontra-se o registro do teste de comportamento em campo aberto das progênies de ratas tratadas com Soyfemme ${ }^{\circledR}$ em duas doses, 4,3 e 43mg/kg, e com Isoflavine ${ }^{\circledR}$ em duas doses, 4,3 e $21,5 \mathrm{mg} / \mathrm{kg}$, e de progênies de ratas dos grupos controle negativo e controle positivo. Os filhotes foram avaliados aos 75 dias

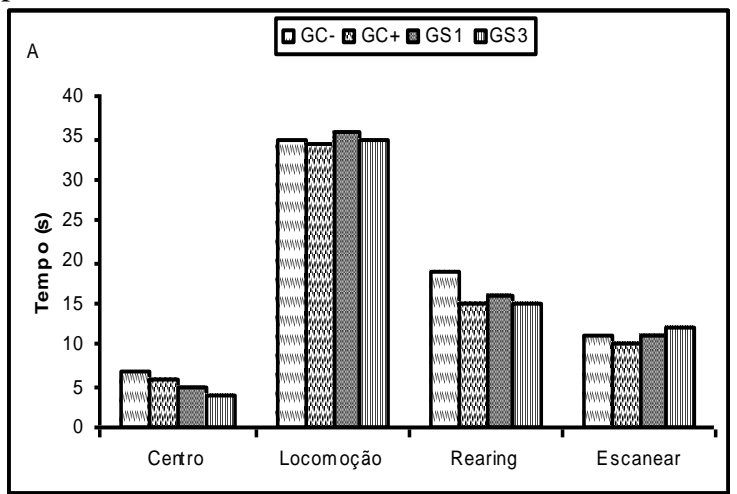

de vida. Durante cinco minutos, foram analisados os parâmetros: frequência de entrada no centro do campo aberto, tempo de locomoção total dentro da caixa, comportamento de "rearing" e comportamento de "escanear". Não houve diferença significativa entre os grupos (ANOVA; $\mathrm{P}>0,05)$.

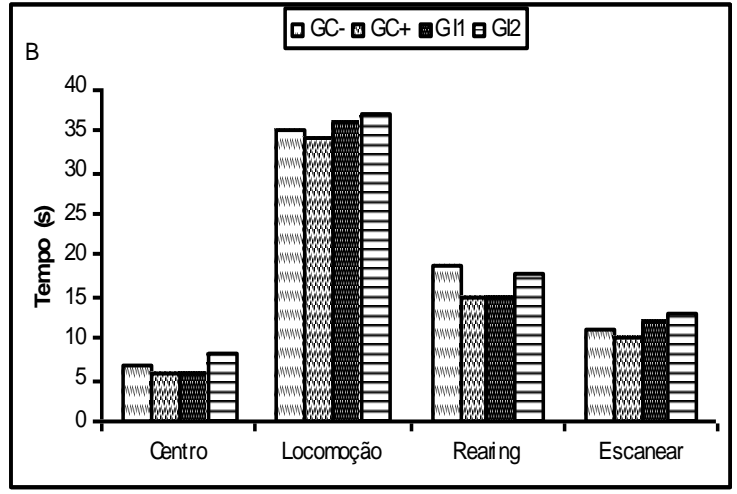

Figura 2. Frequência de entrada no centro do campo aberto, tempo de locomoção total dentro da caixa, comportamento de "rearing" e comportamento de "escanear" da progênie de ratas tratadas com Soyfemme ${ }^{\circledR}(\mathrm{A})$ em duas doses, 4,3 e 43mg/kg, e com Isoflavine ${ }^{\circledR}(\mathrm{B})$ em duas doses, 4,3 e 21,5mg/kg, e de ratas dos grupos controle negativo (GC-) e controle positivo $(\mathrm{GC}+)$.

Na Fig. 3, mostra-se a idade (dias de vida) dos filhotes de ratas tratadas durante os períodos de pré-acasalamento, acasalamento e gestação, no aparecimento das características de desenvolvimento sexual - descida dos testículos, separação prepucial e abertura do canal vaginal. A descida dos testículos ocorreu entre o $15^{\mathrm{o}}$ e o $19^{\circ}$ dia de vida em todos os animais (Fig. 3A), a separação prepucial entre o $34^{\circ}$ e o $39^{\circ}$ dia de vida (Fig. 3B) e a abertura do canal vaginal, entre o $30^{\circ}$ e o $37^{\circ}$ dia de vida (Fig. 3C). Tanto nas características de desenvolvimento geral quanto nas de desenvolvimento sexual, não ocorreu diferença entre os grupos estudados (teste $\chi^{2} ; \mathrm{P}>0,01$ ). 

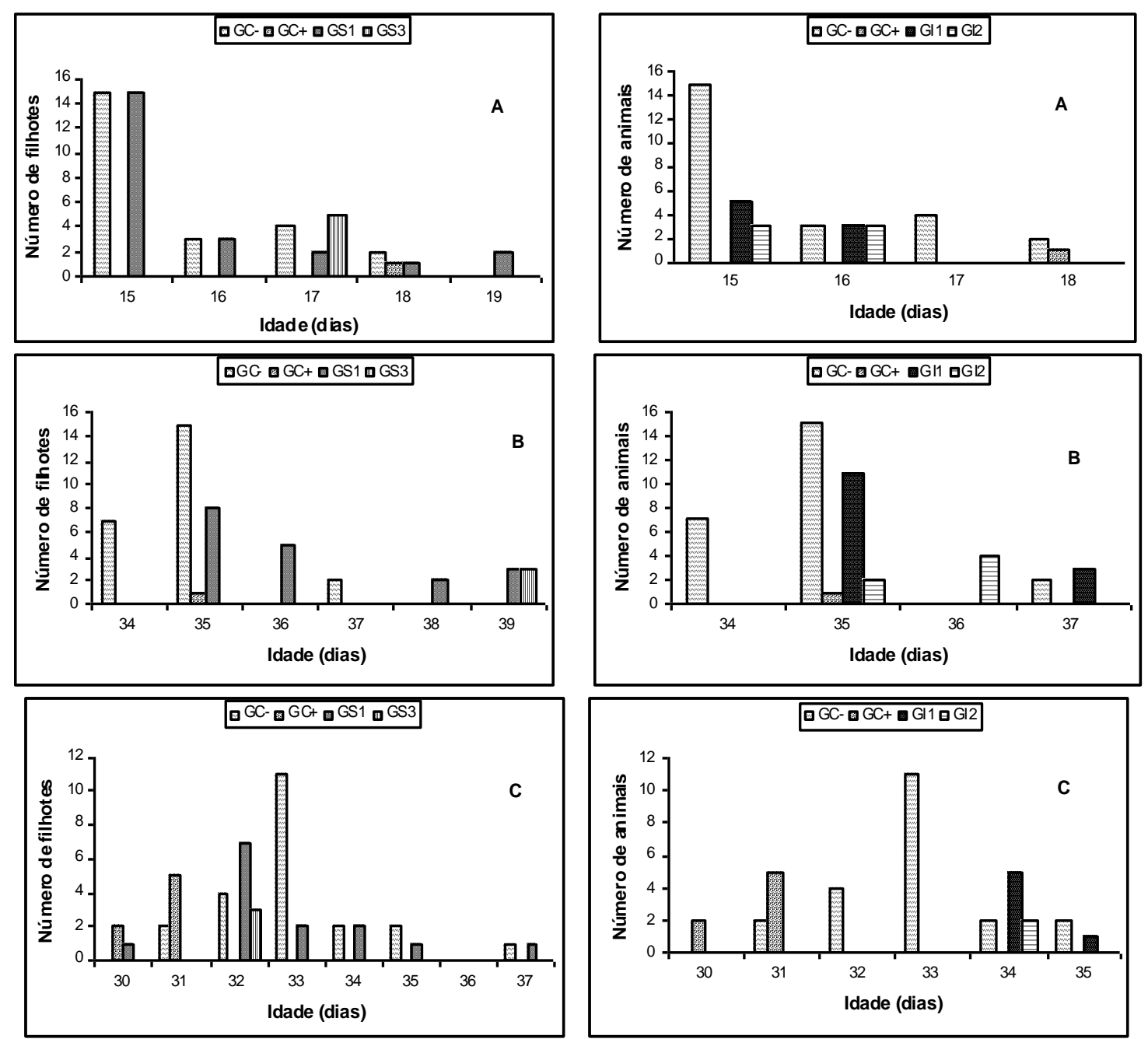

Figura 3. Idade em dias do aparecimento de características de desenvolvimento sexual da progênie de ratas tratadas com Soyfemme (GS) em duas doses, 4,3 e 43mg/kg, e com Isoflavine (GI) em duas doses, 4,3 e $21,5 \mathrm{mg} / \mathrm{kg}$, e progênie de ratas dos grupos controle negativo (GC-) e controle positivo (GC+) durante os períodos de pré-acasalamento, acasalamento, gestação e lactação. A: descida dos testículos; B: separação prepucial; C: abertura do canal vaginal.

\section{DISCUSSÃO}

A exposição pré-natal e a pós-natal a tóxicos podem produzir mudanças que não podem ser previstas a partir dos efeitos observados em adultos, e esses efeitos são muitas vezes irreversíveis. Resultados adversos no desenvolvimento, em ambos os sexos, podem resultar de exposição a agentes tóxicos no útero ou no leite, segundo a Environmental Protection Agency (Guidelines..., 1996). Muitos desses efeitos tóxicos têm sido detectados em homens e mulheres expostos no período pré-natal com estrogênios, progesteronas, andrógenos e antiandrógenos (Giusti et al., 1995; Harrison et al., 1995).

O período de gestação é uma das fases mais sensíveis do ciclo reprodutivo e que resulta em repostas importantes. Hoje, sabe-se que, nesse período, a maioria dos agentes atravessa facilmente a placenta e, dessa maneira, pode-se considerar que a exposição materna a agentes externos, entre esses os agentes químicos, pode resultar em efeitos importantes sobre um organismo passivo, alvo secundário desses agentes, que é o organismo embriofetal (Damasceno et al., 2008). 
Os resultados sugerem que o desenvolvimento pós-natal da prole de ratos Wistar não foi afetado pelo tratamento com as preparações fitoterápicas que continham soja, nas fases de préacasalamento, acasalamento, gestação e lactação em três diferentes doses utilizadas. O tratamento das progenitoras durante todo o período gestacional não determinou aumento no percentual de alterações ósseas nos fetos.

Atualmente há necessidade de estudos longitudinais no que se refere à toxicologia reprodutiva, abrangendo não só alterações estruturais, mas também modificações funcionais, como o desenvolvimento de características físicas desde os primeiros dias de vida e até estudos de comportamento animal, conforme sugeriu Bernardi (2007).

Ao estudarem a transferência lactacional da isoflavona da soja genisteína, em ratos SpragueDawley que a consumiam, Doerge et al. (2006) sugeriram que a exposição de filhotes de ratos ao longo da lactação produz modestos níveis circulantes de genisteína. Essa baixa exposição interna, aparentemente, é resultado da pequena quantidade secretada para o leite. Os resultados encontrados em humanos relatam que o nível total de genisteína transferida para o leite materno era inferior a $10 \%$ da concentração presente no plasma da mãe (Franke et al., 1998).

A exposição materna e paterna a agentes que podem afetar os processos reprodutivos visa à avaliação de problemas de fertilidade, sendo fundamental a exposição durante os períodos críticos de desenvolvimento embriológico e fetal, que compreendem o período de implantação, organogênese e desenvolvimento fetal. Isso justifica a administração das preparações fitoterápicas à base de soja durante todo o período gestacional.

O estudo do potencial teratogênico das preparações deteve-se na avaliação óssea dos fetos, pois o período crítico desse sistema é bastante longo, e quanto maior for o período de exposição tanto mais suscetível este será aos efeitos do agente (Bernardi, 2007).

Em um estudo do desenvolvimento embrionário e fetal, a genisteína foi administrada por via oral aos ratos, nas doses de $0,20,150$ e $1000 \mathrm{mg} / \mathrm{kg} /$ dia, a partir de 6-20 dias de gestação de fêmeas que amamentaram seus descendentes até ao dia 7 da lactação. Na maior dose testada, foi observado o menor ganho de peso das ninhadas durante o período de lactação. Na dose intermediária de $150 \mathrm{mg} / \mathrm{kg} /$ dia e na dose de $1000 \mathrm{mg} / \mathrm{kg} / \mathrm{dia}$, ocorreu menor média de peso corporal individual ao nascer. Nesse mesmo estudo, avaliou-se o potencial teratogênico da genisteína por via oral, e não se observou nenhum feto malformado na avaliação macroscópica externa. $\mathrm{Na}$ avaliação esquelética, observaram-se anomalias no esterno e costelas e ossificação incompleta (McClain et al., 2007).

O peso corporal dos fetos de fêmeas tratadas com as preparações fitoterápicas, que continham Glycine max (L.) Merr. (soja), não diferiu do peso dos fetos do grupo-controle negativo (Tab. 2). O efeito da exposição pré-natal a essas preparações fitoterápicas quanto à ocorrência de anormalidades do esqueleto fetal foi apresentado na Tab. 3, e não houve diferença significante entre os grupos tratados e o grupo-controle negativo, já que no grupo-controle positivo não houve cesariana. No entanto, a maior incidência de anormalidades observadas em todos os grupos foi a ocorrência de anomalias na coluna vertebral, tais como costelas rudimentares, agenesia de costelas e esterno mal ossificado, variáveis estas que são consideradas como variantes da espécie (Damasceno et al., 2008). Os resultados indicaram que as doses testadas não causaram efeitos teratogênicos aos fetos de ratos Wistar.

Doerge et al. (2001) avaliaram a transferência placentária da isoflavona da soja genisteína, pela administração na dieta de ratos Sprague Dawley. Nesse estudo, sugere-se que a genisteína atravessou a barreira placentária, o cérebro e provavelmente outros órgãos do desenvolvimento fetal do rato. Lamartiniere et al. (2000) relataram baixa concentração de genisteína no soro fetal de ratos, após sua administração via dieta materna.

O teste de campo aberto tem sido amplamente utilizado para quantificar movimentos locomotores e de exploração dos animais de laboratório em experimentos com roedores, e esse comportamento é essencial para compreender o efeito de diferentes drogas estimulantes (Eilan, 2003). Na avaliação realizada com o teste de comportamento em 
campo aberto, cuja finalidade foi medir o nível de ansiedade na prole dos animais tratados e dos animais dos grupos controle, observou-se que a exposição das ratas aos fitoterápicos não alterou a desempenho de seus filhotes.

Sendo assim, é possível afirmar que as doses utilizadas neste experimento, a dose terapêutica recomendada pelos fabricantes dos fitoterápicos e a utilização de cinco e 10 vezes essa dose a ratos Wistar não comprometeram o desenvolvimento geral de sua progênie e também não determinaram efeitos embriotóxicos aos fetos.

\section{REFERÊNCIAS BIBLIOGRÁFICAS}

AZEVEDO, M.S. Efeito de intervenções no ambiente neonatal sobre a relação mãe-filhote $e \quad o$ comportamento na idade adulta. 2005. 100f. Dissertação (Mestrado) - Faculdade de Ciências Biológicas, Universidade Federal do Rio Grande do Sul, Porto Alegre.

BERNARDI, M.M. Exposição aos medicamentos durante o período perinatal. In: Farmacologia aplicada à medicina veterinária. Rio de Janeiro: Guanabara Koogan, 2007. 897p.

CHAHOULD, I.; FAQI, A.S. Atlas of external and skeletal anomalies in rats. Berlin: Institute for Clinical Pharmacology and Toxicology/Leipzig: PR \& C Agentur, 1997. (CD-ROM).

COLIE, C.F. Male mediated teratogenesis. Reprod. Toxicol., v.7, p.3-9, 1993.

DAMASCENO, D.C.; KEMPINAS, W.G.; VOLPATO, G.T. et al. Anomalias congênitas: estudos experimentais. Belo Horizonte: Coopmed, 2008. 102p.

DELCLOS, B.K.; BUCCI, T.J.; LOMAX, L.G. et al. Effects of dietary genistein exposure during development on male and female CD (Sprague Dawley) rats. Reprod. Toxicol., v.15, p.647-663, 2001.

DOERGE, D.R.; CHURCHWELL, M.I.; CHANG, H.C. et al. Placental transfer of the soy isoflavone genistein following dietary and gavage administration to Sprague Dawley rats. Reprod. Toxicol., v.15, p.105110, 2001.

DOERGE, D.R.; TWADDLE, N.C.; CHURCHWELL, M.I. et al. Lactational transfer of the soy isoflavone, genistein, in Sprague-Dawley rats consuming dietary genistein. Reprod. Toxicol., v.21, p.307-312, 2006.

EILAN, D. Open-field behavior withstands drastic changes in arena size. Behav. Brain Res., v.142, p.5362, 2003.
FRANKE, A.A.; CUSTER, L.J.; TANAKA, Y. Isoflavones in human breast milk and other biological fluids. J. Am. Clin. Nutr., v.68, suppl.14, p.66-73, 1998.

GIUSTI, R.M.; IWAMOTO, K.; HATCH, E.E. Diethylstilbestrol revisited: a review of the long-term health effects. Ann. Intern. Med., v.122, p.778-788, 1995.

GUIDELINES for reproductive toxicity risk Assessment. Environmental Protection Agency. Federal Register, v.61, p.56274-56322, 1996.

HARRISON, P.T.C.; HUMFREY, C.D.N.; LICHFIELD, M. et al. Assessment on environmental oestrogens: consequences to human health and wildlife. Leicester, UK: MRC Institute for Environment and Health, 1995.

LAMARTINIERE, C.A.; ZHAO, Y.X.; FRITZ, W.A. Genistein: mammary cancer chemoprevention, in vivo mechanisms of action, potential for toxicity, and bioavailability in rats. J. Women's Cancer, v.2, p.1119, 2000.

LAPPONI, J.C. Estatística usando Excel. São Paulo: Lapponi Treinamento e Editora, 2000. 451p.

LEMÔNICA, I.P. Teratogênese experimental e sua aplicação em humanos. In: SPRITZER, D.T.; SANSEVERINO, M.T.V.; SCHÜLER-FACCINI, L. (Eds). Manual de teratogênese. Porto Alegre: UFRGS, 2001. p.19-39.

McClAIN, R.M.; WOLZ, E.; DAVIDOVICH, A. et al. Reproductive safety studies with genistein in rats. Food Chem. Toxicol., v.45, p.1319-1332, 2007.

MELLO, J.R.B.; LANGELOH, A. Avaliação da toxicidade reprodutiva e teratogenicidade. In: RHODEN, E.L.; RHODEN, C.R. (Eds). Princípios $e$ técnicas em experimentação animal. Porto Alegre: UFRGS, 2006. p.455-464.

ORGANIZATION FOR ECONOMIC COOPERATION AND DEVELOPMENT. The validation of test methods considered for adoption as OECD test guidelines. Paris: OECD, 1998. (ENV/MC/CHEM (98) 6).

TAYLOR, W.R.; VAN DIKE, G.C. Revised procedures for staining and clearing small fishes and others vertebrates for bone and cartilage study. Cybion, v.9, p.107-119, 1985.

WANG, H.; MURPHY, P.A. Isoflavones content in commercial soybean foods. J. Agric. Food Chem., v.42, p.1666-1673, 1994.

ZENICK, H.; CLEGG, E.D. Assessment of male reproductive toxicity: A risk of assessment approach. In: Principles and methods of toxicology. New York: Raven, 1989. p.275-309. 